\title{
Detection of the pandemic norovirus variant GII.4 Sydney 2012 in Rio Branco, state of Acre, northern Brazil
}

\author{
Luciana Damascena da Silva/ ${ }^{+}$, Evandro Leite Rodrigues, Maria Silvia Sousa de Lucena, \\ Ian Carlos Gomes de Lima, Darleise de Sousa Oliveira, Luana Silva Soares, \\ Joana D'Arc Pereira Mascarenhas, Alexandre da Costa Linhares, Yvone Benchimol Gabbay
}

Seção de Virologia, Instituto Evandro Chagas, Ananindeua, PA, Brasil

Noroviruses (NoVs) are important cause of gastroenteritis in humans worldwide. Genotype GII.4 is responsible for the majority of outbreaks reported to date. This study describes, for the first time in Brazil, the circulation of NoV GII.4 variant Sydney 2012 in faecal samples collected from children aged less than or equal to eight years in Rio Branco, state of Acre, northern Brazil, during July-September 2012.

Key words: norovirus - gastroenteritis - GII.4 variant

Noroviruses (NoVs) are the major cause of epidemic viral gastroenteritis and the leading cause of foodborne outbreaks in diverse countries in the world. The disease in most cases occurs with diarrhoea and vomiting, affecting mainly children, the elderly and immunocompromised persons. These viruses are often associated with outbreaks in closed settings such as schools, hospitals, hotels, cruise ships and nursing homes (Green 2007).

The importance of NoV as agents of childhood gastroenteritis extends throughout the developing countries since the late 1970s, being considered the second causative agent of viral gastroenteritis in children under five years old (Rackoff et al. 2013). NoVs possess an icosahedral virion of $27-32 \mathrm{~nm}$ in diameter, single stranded, positive-sense, polyadenylated RNA genome of 7,400-7,700 nucleotides (Kapikian et al. 1972). The viral genome is organised into three open reading frames (ORFs). ORF1 encodes the non-structural proteins, including the RNAdependent RNA polymerase (Clarke \& Lambden 2000). The ORF2 encodes the structural capsid protein VP1, the main component of the viral capsid, divided into three major structural domains: S, P1 and P2. The P2 domain contains the most variable sequence and is located on the surface of the capsid, playing an important role in immune recognition and receptor interaction (Prasad et al. 1999, Glass et al. 2000).

NoVs exhibiting a large genetic diversity and are classified into five genogroups (GI-GV) based on VP1 amino acid sequence and these are further subdivided into multiple genotypes (Zheng et al. 2006). Human infection is associated with GI, GII and GIV genogroups,

doi: 10.1590/0074-0276130293

Financial support: IEC

ELR contributed equally to this work.

+ Corresponding author: lucianasilva@iec.pa.gov.br

Received 31 May 2013

Accepted 16 August 2013 of which the GII.4 genotype has maintained importance over more than 30 years in both outbreaks and sporadic cases (Siebenga et al. 2009, Rackoff et al. 2013). Diverse GII.4 variants have been associated with global epidemics of acute gastroenteritis from 1996 to the present, including the US 1995/96 variant in 1996 (White et al. 2002), Farmington Hills variant in 2002 (Widdowson et al. 2004), Hunter variant in 2004 (Bull et al. 2006), 2006a and 2006b variant in 2007-2008 (Eden et al. 2010), New Orleans variant in 2009-2012 (Yen et al. 2011) and most recently Sydney 2012 (van Beek et al. 2013).

The first report of Sydney 2012 variant was in March 2012 in New South Wales, Australia (van Beek et al. 2013). Thereafter, it was detected in the United States of America (USA) in September 2012 (Barclay et al. 2013), in Belgium, September and December 2012, and in Denmark in November 2012 (Fonager et al. 2013). In this period, various countries, such as New Zealand, France, Scotland, Japan, Hong Kong and the USA, have reported a higher incidence of NoV outbreaks (Barclay et al. 2013, Bennett et al. 2013, Chan \& Chan 2013). According to published data obtained by NoroNet, there are suggestive evidences that this increase is related to this new NoV GII.4 2012 variant (van Beek et al. 2013).

This study analysed 25 stool samples of sporadic cases of acute gastroenteritis among hospitalised children which were obtained within the National Surveillance Program of Rotavirus Gastroenteritis coordinated by the Brazilian Ministry of Health. This program comprises three officials Reference Centres and Evandro Chagas Institute is one of them.

The detection of NoV was first performed using a commercial enzyme immunoassay (EIA) (Ridascreen $^{\circledR}$ Norovirus 3rd Generation, R-Biopharm AG, Darmstadt, Germany) following the manufacturer's instructions. Viral RNA was extracted from a $10 \%$ faecal suspension (the same used in the EIA test) using a guanidine isothiocyanate/silica method (Boom et al. 1990) followed by cDNA synthesis performed using a $\mathrm{pd}(\mathrm{N}) 6^{\mathrm{TM}}$ random primer (Amersham Biosciences, UK) and the Superscript ${ }^{\mathrm{TM}}$ II RNAse H Reverse Transcriptase (Invitrogen, USA). 
Initially, the viral genome was amplified by reverse transcription polymerase chain reaction (RT-PCR) using primers for B region of the polymerase gene (ORF1) (Anderson et al. 2001, Fankhauser et al. 2002). For genetic characterisation were used primers for region D (ORF2) of the viral capsid of NoV GII with primers Cap C, Cap D1 and Cap D3 (Vinjé et al. 2004). In addition, these samples were tested with primers for $\mathrm{P} 2$ region (EVP2F and EVP2R) described to define GII.4 variants (Vega et al. 2011). The amplicons obtained were purified with a QIAquick ${ }^{\circledR}$ PCR Purification Kit and QIAquick ${ }^{\circledR}$ gel Purification Kit (QIAGEN, Valencia, CA, USA) following the manufacturer's recommendations. DNA sequencing was performed using the ABI Prism 3130xl Genetic Analyzer (Applied Biosystems, Foster City, CA, USA). The sequences were edited using the BioEdit Sequence Alignment Editor (v.7.0.9.1) software. The evolutionary history was inferred using the neighbour-joining method, bootstrap 2,000 replicates and model using MEGA 5.1 (Saitou \& Nei 1987, Tamura et al. 2011).
From July-September 2012, 25 samples were collected by the Surveillance Program from children with acute gastroenteritis the city of Rio Branco, state of Acre. Patient's ages ranged from four month-eight years $(40 \%$, $0-12$ months; $24 \%, 1-2$ years old; $28 \%>2$ years old; $8 \%$ no information). These samples were analysed by EIA and RT-PCR using region B with a positivity of $48 \%$ $(12 / 25)$ in both, but one sample was positive only by EIA and another only by RT-PCR. All positive cases were shown to have diarrhoea as the major symptom, with duration of four days on average; there were no other symptom recorded on medical histories sent to us.

NoVs genotypes were characterised in four samples using primers for the capsid region (region D). The New Orleans 2009 was detected in one sample and Sydney 2012 GII.4 variants in three samples. The analyses of P2 region confirmed the presence of the Sydney 2012 GII.4 variant and the sequences obtained had a high nucleotide identity among them (range 98.8-99.5\%) and differed in the range of $1.2-2.6 \%$ when compared with the prototype
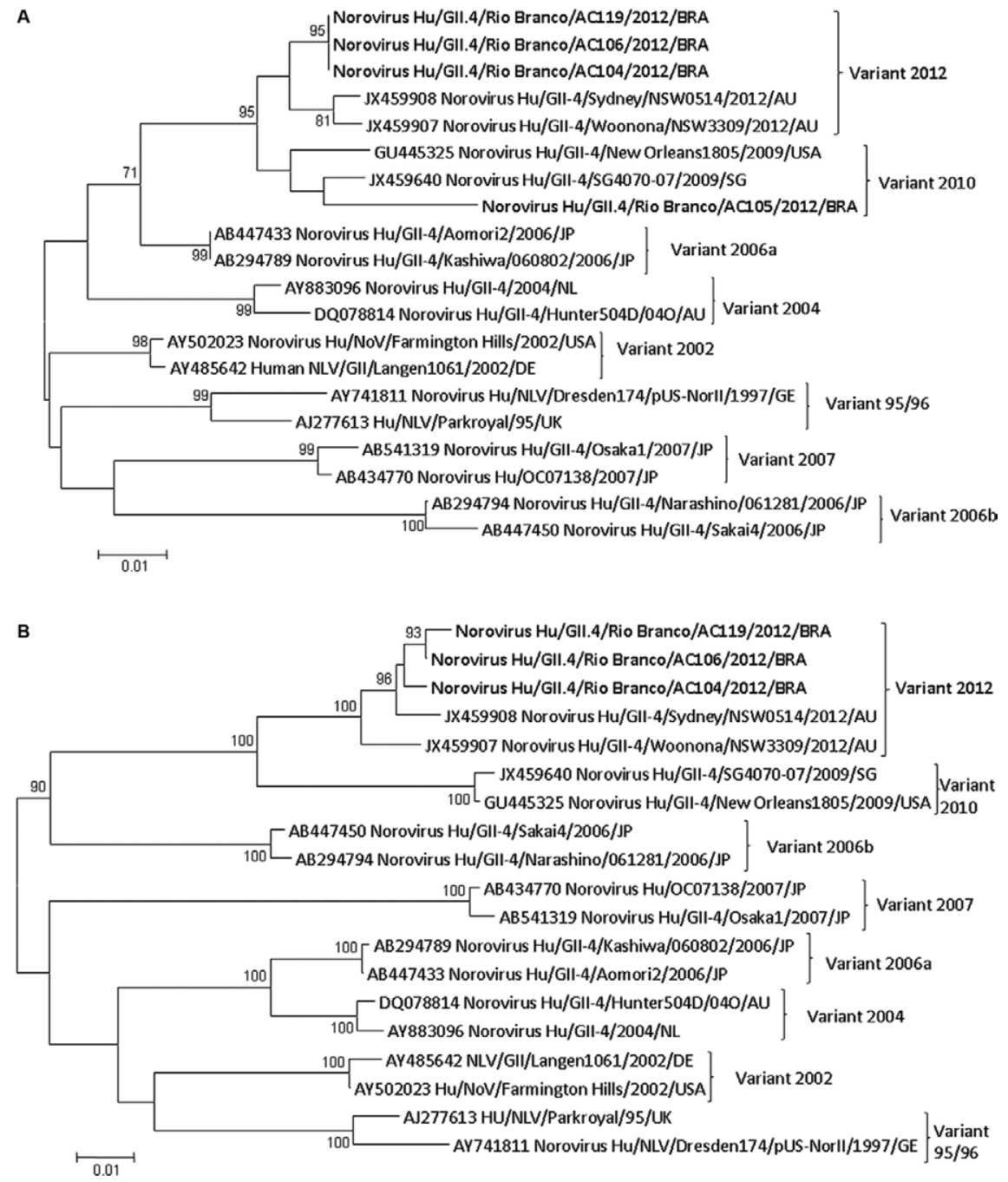

Dendograms constructed using region D (A) and P2 region (B) amplified from strains from diarrhoeic children of the city of Rio Branco, state of Acre, Brazil. Prototype strains are presented. Study samples were marked in bold. The number above each branch corresponds to the bootstrap value (2,000 replicates). The scale bar is proportional to the genetic distance. 
strain (Figure). These sequences were submitted to GenBank under accessions KF360222-KF360228.

Briefly, this study demonstrated by phylogenetic analysis the circulation of the two most recently identified GII.4 variants, New Orleans 2009 and Sydney 2012 in Rio Branco and, to our knowledge, this represents the first detection of the recently emerged GII.4 Sydney 2012 variant in Brazil.

Several studies demonstrate that Sydney 2012 variant has the potential for strain replacement and can rapidly diversify within the population, a phenomenon driven by evolutionary forces and leading to persistence of the GII.4 variants in a community (Eden et al. 2010, Fonager et al. 2013). As a consequence, a rapid worldwide dispersion of the GII.4 variants may occur; nevertheless, further studies are needed in order to better assess this issue, including its association with gastroenteritis outbreaks.

Our results warrant additional studies in other settings in Brazil to see whether this variant is spreading across the country.

In addition, further local studies are needed, including a larger sample size to better evaluate epidemiological and molecular aspects related to the emergence of this new GII.4 2012 variant.

\section{ACKNOWLEDGEMENTS}

To the support provided by Dielle Monteiro Teixeira, Euzeni Menezes and all students and technical of the Norovirus and Astrovirus Laboratory, and to the Health Department of Acre State, for the samples.

\section{REFERENCES}

Anderson AD, Garrett VD, Sobel J, Monroe AS, Fankhauser RL, Schwab KJ, Bresee JS, Mead PS, Higgins C, Campana J, Glass RI 2001. Multistate outbreak of Norwalk-like virus gastroenteritis associated with a common cateter. Am J Epidemiol 11: 1013-1019.

Barclay L, Wikswo M, Gregoricu N, Vinjé J, Lopman B, Parashar U, Hal A 2013. Emergence of new norovirus strain GII.4 SydneyUnited States, 2012. MMWR Morb Mortal Wkly Rep 62: 55.

Bennett S, MacLean A, Miller RS, Aitken C, Gunson RN 2013. Increased norovirus activity in Scotland in 2012 is associated with the emergence of a new norovirus GII.4 variant. Euro Surveill 18: e20349.

Boom R, Sol CJ, Salimans MM, Jansen CL, Wertheim-van Dillen PME, van der Noordaa J 1990. Rapid and simple method for purification of nucleic acids. J Clin Microbiol 28: 495-503.

Bull RA, Tu ET, McIver CJ, Rawlinson WD, White PA 2006. Emergence of a new norovirus genotype II.4 variant associated with global outbreaks of gastroenteritis. J Clin Microbiol 44: 327-333.

Chan MC, Chan PK 2013. Complete genome sequence of a novel recombinant human norovirus genogroup II genotype 4 strain associated with an epidemic during summer of 2012 in Hong Kong. Genome Announc 1: e00140-12.

Clarke IN, Lambden PR 2000. Organization and expression of calicivirus genes. J Infect Dis 181 (Suppl.): S309-S316.

Eden JS, Bull RA, Tu E, McIver CJ, Lyon MJ, Marshall JA, Smith DW, Musto J, Rawlinson WD, White PA 2010. Norovirus GII.4 variant $2006 \mathrm{~b}$ caused epidemics of acute gastroenteritis in Australia during 2007 and 2008. J Clin Virol 49: 265-271.

Fankhauser RL, Monroe SS, Noel JS, Humphrey CD, Bresee JS, Parashar UD, Ando T, Glass RI 2002. Epidemiological and molec- ular trends of "Norwalk-like viruses" associated with outbreaks of gastroenteritis in the United States. J Infect Dis 186: 1-7.

Fonager J, Hindbak LS, Fischer TK 2013. Rapid emergence and antigenic diversification of the norovirus 2012 Sydney variant in Denmark, October to December, 2012. Euro Surveill 18: 20413.

Glass PJ, White LJ, Ball JM, Leparc-Goffart I, Hardy ME, Estes MK 2000. Norwalk virus open reading frame 3 encodes a minor structural protein. J Virol 74: 6581-6591.

Green KY 2007. Caliciviridae: the noroviruses. In BN Fields, Fields virology, 5th ed., Lippincott, Williams \& Wilkins, Philadelphia, p. 949-979.

Kapikian AZ, Wyatt RG, Dolin R, Thornhill TS, Kalica AR, Chanock RM 1972. Visualization by immune electron microscopy of a 27 $\mathrm{nm}$ particle associated with acute infectious nonbacterial gastroenteritis. J Virol 10: 1075-1081.

Prasad BV, Hardy ME, Dokland T, Bella J, Rossmann MG, Estes MK 1999. X-ray crystallographic structure of the Norwalk virus capsid. Science 286: 287-290.

Rackoff LA, Bok K, Green KY, Kapikian AZ 2013. Epidemiology and evolution of rotaviruses and noroviruses from an archival WHO Global Study in Children (1976-79) with implications for vaccine design. PLOS ONE 8: e59394.

Saitou N, Nei M 1987. The Neighbor-joining method: a new method for reconstructing phylogenetic trees. Mol Biol Evol 4: 406-425.

Siebenga JJ, Vennema H, Zheng DP, Vinje J, Lee BE, Pang XL, Ho ECM, Lim W, Choudekar A, Broor S, Halperin T, Rasool NBG, Hewitt J, Greening GE, Jin M, Duan ZJ, Lucero Y, O'Ryan M, Hoehne M, Schreier E, Ratcliff RM, White PA, Iritani N, Reuter G, Koopmans M 2009. Norovirus illness is a global problem: emergence and spread of norovirus GII.4 variants, 2001-2007. J Infect Dis 200: 802-812.

Tamura K, Peterson D, Peterson N, Stecher G, Nei M, Kumar S 2011. MEGA5: molecular evolutionary genetics analysis using maximum likelihood, evolutionary distance, and maximum parsimony methods. Mol Biol Evol 28: 2731-2739.

van Beek J, Ambert-Balay K, Botteldoorn N, Eden JS, Fonager J, Hewitt J, Iritani N, Kroneman A, Vennema H, Vinjé J, White PA, Koopmans M 2013. NoroNet indications for worldwide increased norovirus activity associated with emergence of a new variant of genotype II.4, late 2012. Euro Surveill 18: 8-9.

Vega E, Barclay L, Gregoricus N, Williams K, Lee D, Vinjé J 2011. Novel surveillance network for norovirus gastroenteritis outbreaks, United States. Emerg Infect Dis 17: 1389-1395.

Vinjé J, Hamidjaja RA, Sobsey MD 2004. Development and application of a capsid VP1 (region D) based reverse transcription PCR assay for genotyping of genogroup I and II noroviruses. $J$ Virol Methods 116: 109-117.

White PA, Hansman GS, Li A, Dable J, Isaacs M, Ferson M, McIver CJ, Rawlinson WD 2002. Norwalk-like virus 95/96-US strain is a major cause of gastroenteritis outbreaks in Australia. J Med Virol 68: 113-118.

Widdowson MA, Cramer EH, Hadley L, Bresee JS, Beard RS, Bulens SN, Charles M, Chege W, Isakbaeva E, Wright JG, Mintz E, Forney D, Massey J, Glass RI, Monroe SS 2004. Outbreaks of acute gastroenteritis on cruise ships and on land: identification of a predominant circulating strain of norovirus - United States, 2002. J Infect Dis 190: 27-36.

Yen C, Wikswo ME, Lopman BA, Vinje J, Parashar UD, Hall AJ 2011. Impact of an emergent norovirus variant in 2009 on norovirus outbreak activity in the United States. Clin Infect Dis 53: 568-571.

Zheng DP, Ando T, Fankhauser RL, Beard RS, Glass RI, Monroe SS 2006. Norovirus classification and proposed strain nomenclature. Virology 346: 312-323. 